\title{
Diderot, Marx und der Aufbruch ins bürgerliche Zeitalter
}

Revolutionen bedürfen einer materiellen Grundlage: sie müssen die Bedürfnisse der Menschen verwirklichen. Diderot hat Marx' Gedanken antizipiert, die Encyclopédie hat er als revolutionäres Unternehmen verstanden, dazu bestimmt, die Macht von Tyrannen, Fanatikern, Unterdrückern und Intoleranten zu brechen. ${ }^{1}$ Seine politischen Ansichten hat er im Eifer des Gefechts gewonnen, als kritischer Beobachter darauf Bezug genommen und es unterstützt. ${ }^{2}$ Wissenschaft, Technik, Handwerk, Landwirtschaft, Industrie, Handel und Produktion galt es zu analysieren, um aus der ökonomischen Basis der Gesellschaft - ganz im Sinne frühliberaler Auffassungen - die Kriterien guter Politik herzuleiten. ${ }^{3}$

Der Titel des Bandes, in dem dieser Beitrag erscheint, lautet Formen des Politischen. Damit sind meines Erachtens rhetorische und ästhetische Stilformen gemeint, die der Formulierung eines politischen Anliegens Nachdruck verleihen. Stilformen eindeutig einem politischen Anliegen zuzuordnen ist nicht einfach, zumal im Fall von Diderot. In seinen Äußerungen verschmelzen viele Stimmen: Grimm, Holbach, Galiani, Raynal, Quesnay, Jaucourt, Saint-Lambert und andere tragen dazu bei. ${ }^{4}$ Hinzu kommt die laut Galiani in der >Sekte< von Diderot gepflegte Kunst, leere Zeilen zu lesen und zu verstehen, was nicht für die Ohren der Zensur bestimmt ist. ${ }^{5}$ Im Folgenden möchte ich zeigen, wie Diderot politisches Wissen aus der Beobachtung des unmittelbaren

1 Denis Diderot: Lettre à Sophie Volland, 26 septembre 1762, in: ders.: Correspondance, édité par Georges Roth, Paris 1955-1970, Tome IV, S. 172. Hier und im Folgenden wird mit der Sigle Corr und Seitenzahl verwiesen auf diese Ausgabe. Zum politischen Denken Diderots immer noch empfehlenswert: Henri Lefebvre: Diderot ou les Affirmations fondamentales du matérialisme, Paris 1949/1983; Jacques Proust: Diderot et l'Encyclopédie, Paris 1962/1995; Elisabeth de Fontenay: Diderot ou le matérialisme enchanté, Paris 1981; Yves Benot: Diderot, de l'athéisme à l'anticolonialisme, Paris 1981.

2 Proust: Diderot (wie Anm. 1), S. 350.

3 Urs Marti-Brander: Die Freiheit des Karl Marx, Berlin 2018, S. 162-182.

4 Jean Starobinski: Diderot, un diable de ramage, Paris 2012, S. 58-82.

5 Ferdinando Galiani: Lettres de l'Abbé Galiani à Madame d'Epinay, Paris 1881, S. 138; Galiani à M. Suard, 8 septembre 1770. 
Geschehens gewinnt und zum Chronisten des sozialen Wandels wird. Jahrzehnte später wird Marx sich bemühen, die Bewegungsgesetze von Wirtschaft und Gesellschaft zu verstehen.

Die Herrschaft der Natur und das Elend der Ideologie

Welche ökonomische Ordnung ist den Bedürfnissen und Fähigkeiten der Menschen angemessen? Die Antwort gibt Diderot mit dem 1751 in der Encyclopédie publizierten Artikel Agriculture. ${ }^{6}$ Die Landwirtschaft ist die nützlichste, wesentlichste der von Menschen erfundenen Künste. Die ersten Menschen, ehrwürdig aufgrund ihrer Sitten, ihrer Herzensgüte, ihrer erhabenen Gefühle, widmeten sich vorzugsweise der Kultivierung der Erde. Fürsten haben ihre Gärten bepflanzt, Gesetzgeber in der Sorge um den Landbau ihre Aufgabe erkannt, Feldarbeit galt als Beweis von Tugend und Unschuld. Politiker und Heerführer waren Bauern, das Bauerntum der angemessene Status freier Bürger, der Reichtum verdankte sich den Früchten der Erde. Sobald der Geist der Eroberung größere Gesellschaften schuf, sobald er Luxus, Handel und alle Zeichen der Bosheit der Völker förderte, verlor die Landwirtschaft ihre Würde. Metalle repräsentierten fortan den Reichtum, die Feldarbeit wurde Söldnern und Sklaven überlassen. Diderot idealisiert eine vergangene Epoche, er entwirft eine Ökonomie, die den Fähigkeiten und Bedürfnissen der Menschen angemessen ist, als ethisch-politische Basis der Gesellschaft taugt, den arbeitenden Menschen adelt, die Arbeitsteilung zwischen Herren und Sklaven aufhebt sowie der Korruption vorbeugt. Der Prozess der Zivilisation ist ein mächtiger Korruptionsfaktor. Geld, Handel, Luxus, Arbeitsteilung, Kolonisierung unterminieren die gute Ordnung, der Hunger nach Reichtum trennt die Klassen. Diderot strebt eine Gesellschaft an, worin jeder Mensch Arbeiter, Bürger und Politiker ist, ein universelles, freies, zur Selbstverwirklichung fähiges Wesen, wie Marx später definieren wird. Doch ein unaufhaltsamer Fortschritt entfernt sich vom Ideal. Gibt es eine Politik, die ihn aufzuhalten vermag? In der physiokratischen Lehre glaubt

6 Denis Diderot: Euvres complètes, édition Hermann, Paris 1975 ff., Tome V, S. 290311. Hier und im Folgenden wird mit der Sigle OC und Seitenzahl verwiesen auf diese Ausgabe. 
Diderot die Antwort gefunden zu haben: Die >Herrschaft der Natur begründet den Vorrang der Landwirtschaft.

François Quesnay, der Begründer der physiokratischen Schule, verfasst 1756 für die Encyclopédie den Artikel Evidence. ${ }^{7}$ Darunter versteht er die unbestreitbare Gewissheit, verleiht dem Begriff aber zusätzlich eine moralisch-theologische Bedeutung. Menschen müssen ihr Verhalten regeln, dem Weisen gelingt dies spontan, während die animalische Freiheit der unvernünftigen, instinktgeleiteten Masse der Zähmung durch Herrschaft und Sanktionsmacht bedarf. Die moralische Freiheit ist die Verpflichtung gegenüber dem göttlichen Gebot. Wenn allerdings die Evidenz nur einer intellektuellen Elite zugänglich ist, ist sie offenkundig nicht evident und um ihre soziale Funktion steht es schlecht. 1767 liest Diderot Lemercier de la Rivières L'Ordre naturel et essentiel des sociétés politiques. Er ist begeistert, bisher habe noch niemand so deutlich erkannt, dass die Ordnung der Gesellschaft vorgegeben sei durch jene der Natur. Niemand habe so klar bewiesen, dass Gesetzgebung darin bestehe, Eigentum zu fördern oder zu bekämpfen. Eine erstaunliche Aussage; Diderot konnte nicht entgangen sein, dass seit Bodin bis zu Locke und Rousseau die Eigentumsfrage im Zentrum des politischen Denkens steht. Lemerciers Buch zieht er gar Montesquieus De l'esprit des lois vor. Niemand habe bislang erkannt, dass die Evidenz die Gegenkraft zur Tyrannei bilde. Erst dies Buch habe ihm die Grenzen der Freiheit vor Augen geführt und ihn überzeugt, in der besten Regierung müssten öffentliche Evidenz und allgemeiner Nutzen herrschen (Corr VII, 75-80). Wie kann Evidenz herrschen, wie kann der Kämpfer für Freiheit und Anhänger von Epikur eine Theorie gutheißen, die soziale Beziehungen auf unveränderliche Gesetze zurückführt? 1767 ist Diderot auf dem besten Weg, sich in den Fallstricken einer fragwürdigen Ideologie zu verfangen. Jeder Mensch hat laut Lemercier ein exklusives Recht auf sich sowie das von ihm Erworbene und Erarbeitete. Klagen über die ungleiche Verteilung des Wohlstands hält er für unzulässig, resultiere doch das Eigentum eines Menschen aus seinen Fähigkeiten. Wer gleiche Bedingungen schaffen wolle, zerstöre Eigentum und Gesellschaft. Diderot plädiert dagegen für eine ausgewogene Verteilung der Reichtümer. Lemercier versteht unter Freiheit, Locke

7 Jean-Baptiste le Rond d'Alembert, Denis Diderot: Encyclopédie ou Dictionnaire raisonné des Sciences, des Arts et des Métiers, Nouvelle impression en facsimilé de la première édition de 1751-1780, Volume 6, S. 146-157. 
folgend, den ungestörten Genuss des Eigentums, was für Diderot allzu bescheiden tönen muss. Die Prinzipien der Gesellschaft sind unveränderlich, wie Lemercier unentwegt predigt; die Menschen haben kein Recht, die Ordnung zu gestalten und zu verändern. Die Evidenz wird zur legislativen Gewalt, die Monarchie zur Garantin der ökonomischen Freiheit. Gott ist höchster Gesetzgeber, der Monarch übt als sein Abbild einen >despotisme légak aus, er verbindet persönliche mit legaler Autorität, da er als privater Eigentümer Frankreichs dem Land das Gesetz gibt. Eine Gewaltenteilung, die dem einfachen Volk politische Kompetenz zugesteht, wird verworfen. ${ }^{8}$ Sind die Gesetze der Wirtschaft identisch mit jenen der Natur, erübrigen sich staatliche Interventionen, so die Grundthese der neuen Wissenschaft des >laissez-fairer. Der >despotisme légak ist unfehlbar, die Ungleichheit der Bedingungen naturgewollt, die Demokratie gefährlich, weil sie dem Volk der Besitzund Vernunftlosen legislative Kompetenz zugesteht.

1756/57 publiziert Quesnay in der Encyclopédie zwei ökonomische Artikel, 1776 wird sein Schüler Turgot als Minister gestürzt. In diesem Zeitraum dominieren die Physiokraten die ökonomischen Debatten und propagieren ihre Reformprojekte. Sie kritisieren die Steuerfreiheit des Adels und den `luxe de décoration<, den ökonomisch schädlichen Luxus der parasitären Feudalklasse, während sie dem arbeitenden Volk das Recht auf den >luxe de subsistance zugestehen. Sie regen die Vereinfachung des willküranfälligen Steuersystems an, Handelsfreiheit, die Aufhebung der Zünfte und der Fronarbeit. Nicht Handel und Industrie, nur die Landwirtschaft schafft Mehrwert, so lautet ihr Credo. 1763 wird der freie Warenverkehr innerhalb Frankreichs, 1764 die Ausfuhr von Getreide zugelassen. Doch die Natur widerlegt ihre Apologeten, auf sie ist kein Verlass, wie Galiani gemahnt hatte, ${ }^{9}$ zwischen 1764 und 1770 kommt es zu Hungersnöten, Aufständen und schließlich zum Ende des Freihandels. Turgot, 1774 zum Finanzkontrolleur ernannt, will die Staatsfinanzen mittels Aufhebung von Adelsprivilegien sanieren, was auf massiven Widerstand stößt. Er führt den freien Handel für Getreide wieder ein, muss angesichts schlechter Ernten und Hungerrevolten 1776 aber zurücktreten. Diderots Sympathie für die Physiokraten weicht seit 1770 einer nüchternen Einschätzung. Zu die-

8 Lemercier de la Rivière: L’ordre naturel et essentiel des sociétés politiques (1767), Paris 2001, S. 24-29, 45, 56-60, 70ff., 150-153, 188ff., 466.

9 Ferdinando Galiani: Dialogues sur le commerce des bleds, Londres 1770, S. 235. 
sem Zeitpunkt erreicht die Finanzkrise einen Höhepunkt, eine Krise, die schließlich zur Revolution führen wird.

Diderots Verteidigung des Evidenzdogmas ist schwer verständlich. Es ärgert ihn, dass Grimm nicht einstimmt in sein Loblied auf den >neuen Solon< Lemercier (Corr VII, 84). Grimm bringe mit der Evidenz die Wahrheit in Verruf und setze das Handwerk des Philosophen herab. Er selbst, Diderot, wolle schlicht ein guter Mensch sein statt eines sublimen Schwätzers (ebd., 170f.). ${ }^{10}$ Im Salon de 1767 wirft er Grimm vor, er habe den Fürsten eine Satire gegen die Evidenz geschickt, ihnen eingeredet, Leidenschaften und private Interessen regierten die Welt, die Philosophen seien nutzlose und zudringliche Schwätzer und das Handwerk des Schusters wertvoller als jenes Montesquieus (OC XVI, 111f.). ${ }^{11}$ Er vergisst, dass er selbst Lemercier Montesquieu vorgezogen hat. Grimm wehrt sich: Er habe keine ssatire de l'évidence< verfasst, wohl aber sich mokiert über ökonomische Scharlatane, die das Wort évidence als Allheilmittel anbieten. Was die Leidenschaften und Interessen betrifft, so regierten sie zwar nicht die Welt, eine seriöse politische Wissenschaft müsse sich aber damit befassen (ebd., 510f.).

Grimm hat in der Correspondance littéraire die ssecte des économistes< scharf kritisiert und den Publikationen zu wirtschaftlichen und politischen Themen jede positive Wirkung abgesprochen. Einzig die Regierung hält er für ein taugliches Instrument des gemeinsamen Wohls oder öffentlichen Übels, sie müsse nur ein gerechtes Steuersystem einführen. Den Physiokraten wirft er vor, die Agrikultur zur mystischen Wissenschaft zu erheben, er sieht in ihnen die Theologen einer neuen Religion, ihr Unternehmen widerspricht dem Geist der Aufklärung; der >despotisme légal ist eine bloße Variante der absoluten Monarchie. ${ }^{12}$ Vernichtend ist Grimms Rezension von Lemerciers L'Ordre naturel et essentiel des sociétés politiques: Es handle sich um ein schlechtes Buch, worauf das Publikum nur aufmerksam geworden sei, weil Diderot es gelobt habe, wie er maliziös bemerkt. Diderot vermöge exzellente Werke exzellent zu beurteilen, schlechte Werke verbessere er

10 Lettre à Madame d'Epinay, Octobre 1767.

11 Was die souverains betrifft: die Correspondance war adressiert an einen Souverain d'Allemagne.

12 Denis Diderot, Friedrich Melchior Grimm etc.: Correspondance littéraire, philosophique et critique, Paris 1879, I/7, S. 429-437. Hier und im Folgenden wird mit der Sigle Corr2 und Seitenzahl verwiesen auf diese Ausgabe. 
und finde darin seine Ideen, nicht jene des Autors (Corr2, 443-450). ${ }^{13}$ Später gibt Diderot sich versöhnlich: er verteidige nicht das Dogma, die physiokratische Propaganda habe eine freiere Diskussionskultur ermöglicht und die Menschen gelehrt, sich mit Fragen von Finanz, Handel, Landwirtschaft, Gesetzgebung und Politik zu befassen. Der öffentliche Streit trage zur Aufklärung bei und fördere eine vernünftige Politik. Möge die Schule überleben, wenn auch ihre Anhänger so ignorant und schwatzhaft sind, wie Galiani und Grimm behaupten. Diderot hat sich elegant aus der Affäre gezogen, unüberhörbar distanziert er sich von der physiokratischen Lehre und formuliert nebenher ein wesentliches Element der Demokratie: die öffentliche Deliberation (Corr IX, 210ff.). ${ }^{14}$

Der Luxus und die Tugend

Ein zentrales Thema der Gelehrtenwelt war der Zusammenhang zwischen Kunst, Reichtum und Luxus. Allgemein wird angenommen, die Künste verdankten ihre Entstehung dem Reichtum, dieser bewirke jedoch deren Vervollkommnung wie ihren Niedergang, wie Diderot im Salon de 1767 konstatiert. Mit Grimm formuliert er ein politisches Programm, das die Frage nach dem guten Luxus beantwortet: Der kluge Fürst widmet sich der Landwirtschaft, statt die Wucherer zu stärken. Er schafft die Institution der Generalsteuerpächter ab. Aller Reichtum verdankte sich unter diesem Regime der Landwirtschaft und vermöchte den größten Luxus zu erzeugen, vorausgesetzt, die Ursachen der Korruption werden überwunden: die Käuflichkeit der Ämter, die Verdrängung der Landwirtschaft durch die Industrie, die Regulierung des Handels, der Machtmissbrauch, die ruinösen Staatsanleihen und die unsinnige Ansammlung großen Vermögens. Es ist für eine Gesellschaft verhängnisvoll, wenn das Geld zum Maß aller Dinge wird, die

13 Vgl. Diderot, Grimm: Correspondance littéraire, S. 1-14. Hier und im Folgenden wird mit der Sigle Corr1 und Seitenzahl verwiesen auf diese Ausgabe. Marx folgt Grimm in der Kritik der Physiokraten; er wundert sich, wie die Enzyklopädisten sie unterstützen konnten, ist doch der ökonomische Liberalismus auf einen autoritären Staat angewiesen; vgl. Marx-Engels Gesamtausgabe, Bd. II/2.2, S. 347, 361. Hier und im Folgenden wird mit der Sigle MEGA und Seitenzahl verwiesen auf diese Ausgabe.

14 Lettre à Grimm, Novembre 1769. 
Habenichtse den Reichtum vorspiegeln müssen und die Arroganz der einen auf die Heuchelei der andern trifft. Der gute Luxus verdankt sich dem realen Überfluss, der schlechte maskiert das Elend und entspringt dem Gegensatz von Reichtum und Misere (OC XVI, 164-169)..$^{15}$

Der Dialog findet seine Fortsetzung in der Satire contre le luxe, à la manière de Perse (OC XVI, 551-557). Die Argumente werden wiederholt: die Käuflichkeit der Ämter und der Machtmissbrauch unersättlicher Steuereintreiber verursachen die Übel der französischen Gesellschaft. Sobald das Gold alle Wege öffnet und alle es begehren, gelten Verdienst und ehrlicher Wetteifer nichts mehr. Wenn Betrüger Reichtum im Überfluss haben, beginnt ein sinnloser und grausamer Kampf zwischen allen Ständen der Gesellschaft. Das Gold wird zum Idol der Nation, es bewirkt, dass einige ihren Reichtum schamlos zur Schau stellen und andere zu Heuchlern werden. In der Gesellschaft gilt das unheilvolle Wort: >Soyons ou paraissons riches`, >Seien oder scheinen wir reich $<$. Die Politik soll die Bauern vom drückenden Gewicht der Steuern befreien, die Ungleichheit der Vermögen vermindern und die Nationen bereichern. Segensreich ist der Reichtum, der die Genüsse vervielfacht und die Künste fördert. Doch der Lauf der Geschichte ist unerbittlich, die Landwirtschaft wird Handel, Industrie und Reichtum fördern, Reichtum lässt die Bevölkerung und die Ungleichheit wachsen. Eine wohlhabende Gesellschaft schätzt Kunst und Wissenschaft nur noch unter dem Aspekt des Nutzens. Tugend und Heldenmut sind nicht mehr zu finden bei den >enfants de l'esprit économique`, den Kindern des ökonomischen Geistes. ${ }^{16}$

Im ersten Text stimmen Diderot und Grimm in der Kritik der bestehenden Verhältnisse überein, in der Satire zeichnet sich ein Dissens ab. Welche Konsequenzen zieht der Kritiker aus der Diagnose? Der Realist hält es für klug, Heimat und Mitmenschen zu schätzen und sich der bestehenden Ordnung der Dinge zu unterziehen. Doch wie soll man glücklich sein inmitten eines angeblich zivilisierten Volkes, das nur nach Gold giert und das Verdienst verachtet, gibt der Pessimist zu bedenken. Handelt es sich um ein Selbstgespräch? Diderot träumt

15 Vgl. Guillaume-Thomas Raynal: Histoire philosophique et politique des établissements et du commerce des Européens dans les deux Indes, Tome I, S. 396-400. Hier und im Folgenden wird mit der Sigle HDI I und Seitenzahl verwiesen auf diese Ausgabe.

16 Zum Zusammenhang von Korruption, Dekadenz und Handel vgl. Galiani: Dialogues (wie Anm. 9), S. 110-114. 
von einer Gesellschaft, worin der Subsistenzluxus für alle Menschen erschwinglich wird, nimmt aber resigniert Kenntnis vom unaufhaltsamen Prozess des Fortschritts. Die Satire soll den Zeitgenossen den Spiegel vorhalten, auf das sie sich bewusst werden, was sie verspielt haben und wie sehr sie sich von trügerischen Erwartungen korrumpieren lassen. Die Kritik wird im Bewusstsein formuliert, dass der Weise auf verlorenem Posten steht. Unsinniger Luxus, Geldgier, ungleiche Verteilung der Reichtümer und die Aufspaltung der Gesellschaft in zwei Klassen gehören zu Diderots wichtigsten Themen (OC XXIV, 384f.; 693ff.; 702).

Politik und Kommerz

Die Lektüre einer Schrift von Ferdinando Galiani hat Diderot dazu bewogen, sein Urteil über die Physiokratie zu revidieren. Galiani konnte zeigen, dass es mit der wissenschaftlichen Qualität dieser Theorie, ihrer Fähigkeit zur Voraussage ökonomischer Entwicklungen nicht weit her war. Entgegen den Voraussagen stiegen die Getreidepreise, die Arbeitslosigkeit nahm zu, die Nachfrage nach anderen Konsumgütern ab, soziales Elend und Hungersnot drohten, die Großgrundbesitzer profitierten. Der Abbé Morellet trat mit seiner Réfutation de l'ouvrage qui a pour titre Dialogues sur le commerce des bleds Galiani entgegen, Diderot rezensierte die Réfutation im Auftrag des Polizeichefs de Sartine kritisch (OC XX, 185-194). ${ }^{17}$ Galiani publizierte die Dialogues sur le commerce des bleds 1770 mit Diderots Unterstützung, der darauf unter dem Titel Apologie de l'abbé Galiani eine Kritik an Morellet verfasste. Galiani hielt die physiokratische Idee, die Landwirtschaft sei Basis des Reichtums, für falsch. Als Produkt des Bodens gehört das Getreide zum Handel und zur ökonomischen Gesetzgebung, als Gegenstand der dringendsten Notwendigkeit und wichtigstes Anliegen der Gesellschaft ist es eine Angelegenheit von Politik und Staatsraison, wie er darlegt. ${ }^{18}$ Die Freiheit der Ausfuhr dient den >monopoleurs< und Wucherern, welche die Preise erhöhen und ganze Provinzen aushungern um sich zu bereichern, schließlich wird es zu Aufständen kom-

17 Vgl. die Einführung von Georges Dulac.
18 Galiani: Dialogues (wie Anm. 9), S. 30. 
men. ${ }^{19}$ Die Ansicht, Eigentum und Freiheit seien die Grundprinzipien der Gesellschaft, stellt Galiani ebenso in Frage; in Krisensituationen überwiegt das Interesse der Gemeinschaft. ${ }^{20}$ Schließlich parodiert er die Rhetorik der Physiokraten: »Il a paru évident aux économistes que l'évidence de leur évidence rendrait évident à toutes les nations l'avantage évident de la libre exportation. ${ }^{21}$

Das sakrosankte Recht auf privates Eigentum muss zurücktreten, sobald es um öffentliche Angelegenheiten und den allgemeinen Nutzen geht, wie Diderot im Anschluss an Galiani meint. Er kritisiert nicht das Recht der Bürger auf privates Eigentum, vielmehr die absolutistische Herrschaft, die es jederzeit verletzt (OC XX, 288f., 313). Damit stellt er ein Axiom der Physiokraten in Frage: Der König als oberster Grundherr kann durchaus einen >despotisme illégak ausüben und das Eigentum im Namen der Staatsraison antasten, die wichtigste Voraussetzung der wohlgeordneten Gesellschaft ist daher nicht der Reichtum, sondern die Sicherheit. Das Recht auf Privateigentum ist eine utopische Phantasie (OC XX, 291f., 296ff.). Diderot mahnt, das Gefühl der Menschlichkeit müsse heiliger sein als ein Eigentumsrecht, wogegen immer wieder verstoßen wird und in dessen Namen man Menschen Hungers sterben lässt (OC XX, 331; vgl. S. 307f.). Handelsfreiheit und Monopolbildung werden die Aufspaltung der Gesellschaft in wenige wohlhabende Landwirte und eine Masse mittelloser Arbeiter provozieren (OC XX, 309-313).

Bodin, Locke und spätere liberale Denker haben der politischen Theorie eine neue Richtung gewiesen: der Zweck der Staatsgründung besteht im Schutz des privaten Eigentums. Bürger müssen ihr Eigentum gegenseitig respektieren, niemand darf unverdient Privilegien beanspruchen (OC XX, 307f.). Als >bourgeois révolutionnaire ${ }^{22}$ muss Diderot dem Eigentum, zusammen mit der Freiheit und dem Glück, vorrangige Bedeutung beimessen. Das enzyklopädische Unternehmen beruht auf dem Grundsatz der Verbindung von Freiheit und Eigentum, einem Grundsatz, dem übrigens auch Marx beipflichten wird, wenn er vom selbsterarbeiteten Eigentum spricht. Für die sozialen Konflikte des 19. Jahrhunderts wird die Erfahrung der arbeitenden Bevölkerung

19 Ebd., S. 178-181.

20 Ebd., S. 209-212.

21 Ebd., S. 301: »Den Ökonomen schien evident zu sein, dass die Evidenz ihrer Evidenz allen Nationen den Vorteil der freien Ausfuhr evident erscheinen lässt.«

22 Gerhardt Stenger: Diderot, le combattant de la liberté, Paris 2013, S. 467-495. 
bestimmend, dass bürgerliches Privateigentum oft ein unverdientes Privileg ist und die Mehrheit keine Chance hat, sich Eigentum anzueignen. Diderot war sensibel genug, die mühevolle Situation der Arbeiter zur Kenntnis zu nehmen. Ihre Aktivitäten sind der Gesundheit abträglich, lassen sie vorzeitig altern und obgleich sie nützlich und notwendig sind, werden sie schlecht bezahlt. Was die Arbeiter quält, ist nicht der geringe Lohn, sondern die Ermüdung, die überlange Dauer ihrer Tätigkeit (OC XXIV, 715).

Die Poetik der Ruinen - revolutionäre Phantasien

»Tout porte en soi un germe secret de destruction«(OC XVI, 556). ${ }^{23}$ Diderot war fasziniert vom Motiv der Ruinen, wie der Salon de 1767 bezeugt. Sie wecken in ihm großartige Gefühle, lassen ihn verstehen, dass alles zugrunde geht, nur die Welt bestehen bleibt, nur die Zeit von Dauer ist. Der Mensch als Wanderer zwischen zwei Ewigkeiten ist vergänglich. Ruinen bringen den Menschen die Vergänglichkeit von Staaten und Gesellschaften zu Bewusstsein. Diderot malt sich aus, wie ein Sturzbach alle Nationen mit sich in den Abgrund reißt, während er alleine am Rand steht und die Fluten zerteilt, die sich an seinen Seiten ergießen - ein neuer Moses? Ruinen beweisen, dass Herrschaftsformen nicht ewig und andere Ordnungen möglich sind. Sie lehren die Menschen die Vergeblichkeit ihres Tuns und lassen sie die Verheerungen der Zukunft vorwegnehmen (OC XVI, 335-339; vgl. HDI I, 453).

Die Radikalisierung von Diderots politischen Ansichten dokumentieren mehrere Äußerungen. »Salus populi suprema lex est« - Diderot hält die Devise für falsch, sie sollte lauten: "Salus dominantium suprema lex est« - das Heil der Herrschenden ist höchstes Gesetz, es schützt den Hirten, nicht die Herde, mit dem Unterschied dass >le souverain-pâtre das Vieh nicht zu saftigen Weiden führt, sondern verhungern lässt und direkt zum Schlachthof führt (Corr V, 173). ${ }^{24} 1769$ stößt Diderot auf eine Schrift von Dom Deschamps, der das Ziel der Zivilisation in

23 "Alles birgt in sich den geheimen Kern der Zerstörung.«

24 Lettre à Sophie Volland, 12 novembre 1765. Vgl. Thrasymachos' Kritik an Sokrates' pastoralem Herrschaftsverständnis: Platon: Politeia 343b. 
einer egalitären Gesellschaft ohne Herrschaft und Gesetz sieht. Die menschliche Gattung könne ihr Glück erst finden, wenn es keine Könige, Priester, Magistraten, kein privates Eigentum und keinen Begriff von Laster und Tugend mehr gibt. Diderot gesteht, die Lektüre habe ihm Vergnügen bereitet, unverhofft habe er sich in der Welt wiedergefunden, für die er bestimmt sei. Er spricht von einem schönen Paradox, hält den anarchischen Zustand für >diablement idéak (Corr IX, 245). ${ }^{25}$ Der Fürstin Dashkoff schreibt er, jedes Jahrhundert werde von seinem Geist bestimmt, der Geist ihrer Gegenwart sei jener der Freiheit. Sobald die Menschen wagten, die Festungen der Religion zu erstürmen, gäbe es kein Halten mehr (Corr XI, 20f) ${ }^{26}$ Wer die Majestät des Himmels mit drohendem Blick anschaut, wird nicht zögern die Souveränität der Erde ebenso zu betrachten. Die Kritik des Himmels verwandelt sich in die Kritik der Erde, so wird Marx formulieren (MEGA III/1, 458). Diderot ist überzeugt, Frankreich nähere sich einer Krise, die in Sklaverei oder Freiheit münden werde. Diderot - ein Revolutionär, ein Anarchist, gar Kommunist? Der Schluss wäre voreilig. Das Ideal ist eine teuflische Sache, Diderot rät zu Realitätssinn: jede Macht, die nicht das Glück der Allgemeinheit anstrebt, ist illegitim, doch keine Macht auf Erden erfüllt das Kriterium, mit dem Bestehenden muss man sich wohl oder übel abfinden. Vorsicht ist geboten, die Lektion von Vincennes kann Diderot nicht vergessen.

Was ist unter radikaler Politik zu verstehen? Diderot sieht sich konfrontiert mit zwei gegensätzlichen Positionen, einer utopischen und einer realpolitischen. Realpolitisch argumentiert der englische Maler Ramsay, dessen Brief Diderot übersetzt. Ramsay kritisiert Beccarias Dei delitti e delle pene sowie generell die >Metaphysik des Sozialvertrags. Einen solchen Vertrag sucht man in der Geschichte vergeblich, Menschen sind herrschsüchtig, die Herrschenden müssen ihre Autorität durchsetzen, notfalls mit grausamen Strafpraktiken (OC XX, 407-419). ${ }^{27}$ Diderot kann dieser Ansicht nicht beipflichten, nimmt aber zur Kenntnis, dass eine rein normativ agierende Philosophie manche Fragen offen lässt.

25 Vgl. Benot: Diderot (wie Anm. 1), S. 144.

26 A la princesse Dashkoff, 3 avril 1771.

27 Lettre traduit de l'anglais de M. Ramsay. 
Sturm und Drang nach Kommerz

Marx hat Diderot als Schriftsteller hoch geschätzt, Jacques le fataliste, Le neveu de Rameau und den Salon de 1767 hatte er gelesen, zitierte gerne daraus, mehrere Bände der Correspondance Littéraire in der Auflage von 1813-1814 waren in seiner Bibliothek vorhanden (MEGA IV 32, 301-302). Er hatte Gelegenheit, philosophische, ökonomische, literarische und politische Debatten im Frankreich der zweiten Hälfte des 18. Jahrhunderts zu verfolgen sowie Texte von Diderot, Grimm, Galiani, Raynal und anderen zu lesen. Was die spolitische Form< betrifft, so stehen bei Diderot und Marx zwei Aspekte im Vordergrund: die Kritik der Entfremdung als negativer, der Fortschrittsglaube als affirmativer Kommentar zum historischen Prozess. »Soyons (riches) ou paraissons riches«. Marx nimmt das Motto der Satire auf, spricht von der ästhetischen Form des Schatzes, dem Besitz von Gold- und Silberwaren. Diderot erwähnt die Arbeiterin, die kaum genug verdient, um sich Brot zu kaufen, aber eine goldene Uhr hat. Marx formuliert knapp: Der Schatzbildner opfert dem Goldfetisch seine Fleischeslust. Er macht Ernst mit dem Evangelium der Entsagung (MEGA II/5, 91). Diderots Rhetorik von Sein und Schein, selbst- und fremdbestimmtem Leben hat Marx inspiriert: das Vermögen, das den Status eines Menschen bestimmt, ist nicht mehr die schöpferische Fähigkeit, sondern die Höhe des monetären Eigentums. Diderot verflucht jene, die das Gold zum Idol der Nation erhoben haben. Dies tut Rameaus Neffe. Er nimmt in der sozialen Hierarchie einen bescheidenen Platz ein und repräsentiert doch den wichtigsten Teil von Stadt und Hof, so belehrt er den Philosophen. Er ist eine Sklavennatur, wie er freimütig einräumt, sucht die Leute zu amüsieren (OC XII, 119, 122). Sein höchstes Gut ist Gold, ihm gilt sein Sehnen. Nie käme er auf die Idee, seinen Sohn Maximen der Tugend zu lehren, wer sich daran hielte, würde zum Bettler. Besitzt er ausnahmsweise einen Louis d'Or, stellt er sich vor den Sohn hin, zieht die Münze aus der Tasche, hebt die Augen zum Himmel empor und küsst sie - die Blasphemie ist deutlich (OC XII, 175). Das Gold wird vom Zirkulationsmittel zum Gott in der Welt der Waren, wie Marx schließt (MEGA II/1.1, 146). Er nimmt die Frage des Luxus auf: Der Kapitalist reduziert das Bedürfnis des Arbeiters auf den elendesten Lebensunterhalt, dessen Tätigkeit auf die abstrakteste mechanische Bewegung; er macht ihn zu einem unsinnlichen, bedürfnislosen Wesen, noch der bescheidenste Genuss, die 
geringste spontane Tätigkeit des Arbeiters erscheint ihm als Luxus. Die Nationalökonomie ist keine Wissenschaft des Reichtums, sondern des »Entsagens, des Darbens, der Ersparung, und sie kömmt wirklich dazu, dem Menschen sogar das Bedürfnis einer reinen Luft oder der physischen Bewegung zu ersparen.« Marx reklamiert für das arbeitende Volk den Luxus, Bücher zu lesen, ins Theater, auf den Ball, ins Wirtshaus zu gehen, zu singen, zu malen und zu fechten (MEGA I/2, 420f.). In der Geschichte der Industrie entdeckt er das saufgeschlagene Buch der menschlichen Wesenskräfteく, die menschliche Psychologie, die bislang stets nur in einer Nützlichkeitsbeziehung gefasst wurde (MEGA I/2, 395). Diderots Kritik des Utilitarismus findet hier ihre Fortsetzung. Industrie bedeutet für Marx die Gesamtheit der produktiv-kreativen Fähigkeiten des Menschen. Er nimmt Elemente der Gesellschaftskritik von Diderot auf und übersetzt sie in die Sprache des 19. Jahrhunderts, worin der industrielle Kapitalismus zur dominierenden Wirtschaftsform geworden ist.

Der Kritiker der Entfremdung ist zugleich Fürsprecher bürgerlich-liberaler Anliegen, er versteht die Französische Revolution als Emanzipation des Bürgertums, fordert Handels- und Gewerbefreiheit als Bedingungen des Fortschritts, steht den kommunistischen Sekten skeptisch gegenüber. Zeit seines Lebens hat Marx einen Kommunismus abgelehnt, der Asketismus und Bedürfnislosigkeit predigt und die Individualität sowie die Welt der Bildung und Zivilisation negiert (MEGA I/2, 399). Das Manifest der Kommunistischen Partei ist ein Loblied auf die Taten der Bourgeoisie. Sie hat die Aufklärung radikalisiert, feudale, patriarchalische, idyllische Verhältnisse zerstört, die Religion im eiskalten Wasser egoistischer Berechnung ertränkt und mittels der großen Industrie den Weltmarkt geschaffen. ${ }^{28}$ Marx erinnert an die bürgerlichen Revolutionen in den Niederlanden, England und Frankreich, versteht sie als Kämpfe gegen Absolutismus, Adel und Kirche, Proklamationen der politischen Ordnung für die neue europäische Gesellschaft, als Sieg des bürgerlichen über das feudale Eigentum, der Konkurrenz über die Zunft, der Aufklärung über den Aberglauben, des Fleißes über die Faulheit, des Rechts über das Privileg (MEW 6, 107f.).

Nach dem Sturz Robespierres wird dessen republikanisches Ideal vom Liberalismus verdrängt, die politische Aufklärung verwirklicht sich

${ }^{28}$ Marx-Engels-Werke, Berlin 1956-1990, Bd. 4, S. 464f. Hier und im Folgenden wird mit der Sigle MEW, Bandnummer und Seitenzahl verwiesen auf diese Ausgabe. 
prosaisch. Die von feudalen Banden befreite bürgerliche Gesellschaft bricht »in gewaltigen Lebensströmungen hervor. Sturm und Drang nach kommerziellen Unternehmungen, Bereicherungssucht, Taumel des neuen bürgerlichen Lebens, dessen erster Selbstgenuss noch keck, leichtsinnig, frivol, berauschend ist [...] «. Aufklärung fördert das wissenschaftliche so gut wie das ökonomische und politische Wissen, Marx folgt hierin den Enzyklopädisten. Die Verdrängung des feudalen durch das kapitalistische Eigentum, die Regungen der befreiten Industrie sind Akte der Aufklärung (MEW 2, 130). Anhand der Konkurrenz zwischen Grundbesitz und Kapitalbesitz demonstriert Marx wie ökonomische Gewissheiten ideologische Konflikte provozieren: Der Grundeigentümer sieht im Kapitalisten den Urheber von Konkurrenz, Pauperismus und sozialer Desintegration. Der Kapitalist verweist auf die Wunder der Industrie, weiß sich als legitimer Vertreter der Moderne, beschert der Welt Freiheit, Moral, Bildung und Völkerverständigung. Beide Seiten überschätzen sich (MEGA I/2, 379ff.). Sieht Marx sich gezwungen, Stellung zu beziehen, wie in den Disputen über den Freihandel in England, gerät er in Verlegenheit. Auf die Seite der konservativen Freihandelsgegner will er sich nicht stellen, doch ist ihm klar, dass die Globalisierung des Handels die Völkerfreundschaft keineswegs stärken wird; was die freie Konkurrenz auf nationaler Ebene an Zerstörung angerichtet hat, muss sich auf globaler Ebene wiederholen (MEW 4, 444-458). Kann somit die Schaffung des Weltmarkts nicht als Akt der Aufklärung gelten? Marx hat die Globalisierung als notwendigen Prozess verstanden, die neuen Akkumulationsmethoden des global expandierenden Kapitalismus jedoch in düstersten Farben gemalt: Die Ausbeutung von Gold und Silber in Amerika, die Ausrottung und Versklavung der eingeborenen Bevölkerung, die Zwangsarbeit in den Bergwerken, die Eroberung und Ausplünderung von Ostindien, der Sklavenhandel in Afrika, den Handelskrieg der europäischen Nationen, das Kolonialsystem, die Staatsschulden, den Protektionismus, die Instrumentalisierung der Staatsgewalt zu Profitzwecken (MEGA II/5, 601f.).

Diderot hat den unaufhaltsamen Gang der Geschichte anhand der Ruinen evoziert, Marx - in einer Zeit des beschleunigten Fortschritts nimmt ein Motiv des Neveu de Rameau auf, den fremden Gott, der sich bescheiden auf den Altar setzt, neben den Landesgötzen. Er breitet sich aus, wird stärker, »und an einem hübschen Morgen gibt er mit dem Ellbogen seinem Kameraden einen Schub, und Bauz! Baradauz! Der 
Götze liegt am Boden. ${ }^{29}$ Der fremde Gott ist das Kolonialsystem, das den Profit zum einzigen Zweck der Menschheit erklärt (MEGA II/5, 604), die erbarmungslose Ausbeutung der Erde und der Menschen ist der neue Gottesdienst.

Marx' Thema ist jenes von Raynal und Diderot: die Globalisierung des Handels, der Kolonialismus, die Sklaverei. Wo liegen die Affinitäten? Diderot wie Marx formulieren eine Ethik der Wissenschaft. Marx will nicht die Person des Kapitalisten verantwortlich machen für Verhältnisse, deren Geschöpf er ist; er versteht sich als Wissenschaftler, dem wissenschaftliche Kritik willkommen ist. Diderot respektiert ausschließlich die heilige Wahrheit. Von Leidenschaften und Vorurteilen will er sich befreien, Herkunft und Status vergessen und seinen imaginären Lesern als guter Freund begegnen, seine Seele von Furcht und Hoffnung reinigen. Er will Irrtümer angreifen, nicht Personen. Seine Seele will er von Furcht und Hoffnung reinigen, über dem Globus schwebend will er alles überblicken. Dank dieser Perspektive ist er frei, vermag den Fortschritt zu ermessen, sieht das Aufblühen von Kunst und Wissenschaft, das Ende der Barbarei und die Zivilisierung der Völker. Wem ist dies zu verdanken? Marx wird der Bourgeoisie das Verdienst zuerkennen, Diderot beruft sich auf das Urteil aufgeklärter Männer, die den Fortschritt dem Handel zurechnen (MEGA II/5, 14f.; HDI I, 24). ${ }^{30}$ Die Zivilisationskritik von Diderot und Marx ist ein Protokoll der Verbrechen der Eroberer und Unterdrücker, doch ist sie nicht nur Anklage, vielmehr Analyse jener Prozesse, die dazu geführt haben, dass Menschen und Nationen unter dem Zwang der Konkurrenz stehen und den Profit zu ihrem ausschließlichen Handlungsmotiv machen.

Vermag der Handel die Harmonie zwischen den Völkern, die gerechtere Verteilung der Reichtümer und die Freiheit aller Menschen zu bewirken angesichts der Monopole, diesen Tyrannen der Industrie? (HDI I, 328). Das Werk von Raynal und Diderot dokumentiert, weshalb dies nicht zutrifft. Marx hat dargelegt, wie die Globalisierung die Arbeits- und Lebensbedingungen der Menschen von weltgeschichtlichen Faktoren abhängig macht, wie technische Erfindungen

29 So die Übersetzung von Goethe. Denis Diderot: Rameaus Neffe, Stuttgart 1984, S. 71. Im Original: »Le dieu étranger se place humblement sur l'autel à côté de l'idole du pays; peu à peu, il s'y affermit; un beau jour, il pousse du coude son camarade; et

patatras, voilà l'idole en bas." OC XII, 163
Vgl. auch Fontenay: Diderot (wie Anm. 1). 
Arbeitsplätze vernichten und große Reiche umwälzen (MEW 3, 45f.). Diderot ruft die Völker auf, sich zu erheben, die Pflastersteine aus den Straßen zu reißen. Den englischen Händlern, die sich zu Herrschern aufgeschwungen haben, schreibt er folgende Maxime zu: »Möge mein Land untergehen, Hauptsache ich kann mich bereichern. Alle Orte auf Erden gelten mir gleich. Habe ich eine Region verwüstet und ausgesaugt, wird stets noch eine weitere übrigbleiben, wohin ich mein Gold tragen und friedlich genießen kann" (HDI I, 327-333 [Freie Übersetzung; U.M.]). »Après moi le déluge! Nach uns die Sintflut«, ist der Wahlruf der Kapitalisten, so bringt es Marx auf den Begriff (MEGA II/5, 212).

Marx' Kapitalismuskritik zielt auf die Instrumentalisierung des Individuums. Eine Arbeitsteilung, die individuellen Bedürfnissen und Fähigkeiten nicht gerecht wird, entwertet die Menschenwelt. Der Instrumentalisierung stellt er das Ideal des universellen, freien, sich selbst verwirklichenden Menschen entgegen und prophezeit ein künftiges Zeitalter freier Individualität (MEGA II/1.1, 90f.; II/5, 476f.). Die Perfektibilität, die Freiheit des Menschen sich zu entfalten und die eignen Potentiale zu verwirklichen, ist für Diderot das Kriterium einer guten, humanen Gesellschaft. Die Politik kann die Menschen nicht perfektionieren, wohl aber die Bedingungen schaffen, welche die Entfaltung ermöglichen. Dies setzt eine Gesellschaft voraus, die sich selbst organisiert, die Menschen zur Freiheit erzieht, die Macht der Regierung beschränkt und ein Recht auf Widerstand kennt. ${ }^{31}$

Der Fortschritt der Zivilisation ist eine vieldeutige Angelegenheit, dies war Diderot so gut wie Marx bewusst. In einer 1856 gehaltenen Rede hielt Marx fest: »In unseren Tagen scheint jedes Ding mit seinem Gegenteil schwanger zu gehen." Die Technik vermöchte die menschliche Arbeit zu verringern, lässt sie aber verkümmern und bis zur Erschöpfung auszehren. Die Quellen des Reichtums verwandeln sich zu Quellen der Not. Die Bezwingung der Natur durch den Menschen hat zur Folge, dass der Mensch durch andre Menschen oder durch seine eigne Niedertracht unterjocht wird (MEW 12, 3f.).

Wie steht es um die >Formen des Politischen<? Es fehlt im ausgehenden 18. und frühen 19. Jahrhundert nicht an Utopien und Ideologien, die den Sinn der Geschichte zu kennen beanspruchen. Diderot begeistert

31 Annie Ibrahim: Diderot. Un matérialisme éclectique, Paris 2010, S. 220; Anthony Strugnell: Diderot's politics, The Hague 1973, S. 67f., 183f. 
sich für die Physiokratie und dessen Konzept des legalen Despotismus, er sympathisiert mit dem aufgeklärten Despotismus, der absoluten Monarchie. ${ }^{32}$ Marx ist von der französischen Aufklärung, von Holbach und Helvétius beeinflusst, ebenso von den Junghegelianern. Je mehr Diderot und Marx sich ins Studium der Geschichte vertiefen, desto kritischer beurteilen sie Ideologien, die beanspruchen deren Ziel zu kennen. Die Wissenschaft der Geschichte überwindet die Ideologien. Diderots Ästhetik hat einen präzisen sozialen, ökonomischen und politischen Inhalt, sie bringt die Bestrebungen der aufsteigenden bürgerlichen Klasse zum Ausdruck. ${ }^{33}$ Diderot, der materialistische, revolutionäre Denker nimmt den historischen Materialismus von Marx vorweg. Beide Denker sind kritische Beobachter, Chronisten eines Geschehens, dessen Logik sie mit mehr oder weniger Erfolg zu entschlüsseln suchen. Die Logik ist jene des Konflikts sozialer Klassen, sie gilt es zu verstehen und zu prüfen, unter welchen Bedingungen der Konflikt zu beenden wäre. So bewegt sich denn das Denken von Diderot und Marx zwischen zwei Säulen: Der Kritik der Deshumanisierung sowie dem Bemühen zu verstehen, wie die Menschen versuchen, unter vorgefundenen Umständen ihre eigene Geschichte zu machen.

32 Ibrahim: Diderot (wie Anm. 31), S. 202, 214.

33 Lefebvre: Diderot (wie Anm. 1), S. 9f. 
